\title{
Evaluación de la jerarquía de los valores humanos de Schwartz en la adolescencia: diferencias de género e implicaciones educativas
}

\author{
VÍCTOR ABELLA GARCÍA \\ Universidad de Burgos, Burgos, España
}

FERNANDO LEZCANO BARBERO

Universidad de Burgos, Burgos, España

RAQUEL CASADO MUÑOZ Universidad de Burgos, Burgos, España

\section{RESUMEN}

Schwartz propone la existencia de diez valores distinguibles en diferentes países y culturas. En este estudio se presenta un análisis exploratorio de estos valores en un grupo de adolescentes. La muestra fue de 499 adolescentes: 267 varones y 232 mujeres. Los participantes completaron el cuestionario de valores personales. La jerarquía de valores obtenida indicó mayor preferencia por hedonismo y los valores que componen los tipos trascendencia y apertura al cambio. Los valores menos preferidos fueron los relacionados con los tipos promoción personal y conservación. Se hallaron diferencias significativas en función de sexo en la importancia otorgada a benevolencia, universalismo, conformidad y seguridad. La jerarquía obtenida para los adolescentes en este estudio difiere de la obtenida para adultos en otras investigaciones. Los resultados pueden contribuir al desarrollo de propuestas didácticas encaminadas al aumento de la motivación del alumnado y a reducir el fracaso escolar.

PALABRAS CLAVE

jerarquía de valores humanos; valores, adolescencia; características psicológicas; educación. 


\section{EVALUATION OF THE HIERARCHY OF HUMAN VALUES BY SCHWARTZ IN ADOLESCENCE: GENDER DIFFERENCES AND EDUCATIONAL IMPLICATIONS}

\section{ABSTRACT}

Schwartz proposed ten value dimensions which are discriminated among countries and cultures in his theory of basic human values. This study explores the values proposed in a group of adolescents. The sample was 499 adolescents: 267 men and 232 women. Participants completed the personal value questionnaire. The value hierarchy obtained showed that hedonism and the values included in the higher order types self-trascendence and openness to change were the preferred values. Values related with the higher order types conservation and self-enhancement were less important values for adolescents. Gender significant differences were found in the importance given to universalism, benevolence, conformity and security. Values hierarchy obtained in this study with adolescents differs from others obtained for adults in previous research. Results obtained may contribute to the development of didactic proposals to increase pupil motivation, and to decrease the academic failure.

KEYWORDS

hierarchy of human values; values; adolescence; psychological characteristics; education.

\section{AVALIAÇÃO DA HIERARQUIA DE VALORES HUMANOS DE SCHWARTZ NA ADOLESCÊNCIA: GÊNERO E IMPLICAÇÕES EDUCACIONAIS}

\section{RESUMO}

Schwartz, em sua teoria de valores humanos básicos, propõe dez valores distinguíveis em diferentes países e culturas. Neste estudo, apresenta-se uma análise exploratória dos valores propostos em um grupo de adolescentes. A amostra foi composta de 499 adolescentes: 267 homens e 232 mulheres. Os participantes completaram o questionário de valores pessoais. A hierarquia de valores obtida indicou uma maior preferência por hedonismo e valores que compõem os tipos de ordem superior, transcendência e abertura à mudança. Os valores menos preferidos foram os relacionados com os tipos promoção pessoal e conservação. Diferenças significativas foram encontradas, em relação ao gênero, na importância dada à benevolência, universalismo, conformidade e segurança. A hierarquia dos valores para os adolescentes participantes difere da obtida em adultos. Os resultados podem contribuir para o desenvolvimento de propostas educacionais que visam aumentar a motivação dos alunos e reduzir o insucesso escolar.

PALAVRAS-CHAVE

hierarquia de valores humanos; valores; adolescência; características psicológicas; educação. 


\section{INTRODUCCIÓN}

En los últimos años se ha venido trabajando sobre los valores en la adolescencia y en sociedad actual se habla de la existencia de una crisis de valores en los jóvenes (Casal; Merino; García, 2011). Existe un acuerdo casi unánime por el cual se considera que la crisis económica de los últimos años tiene como causa última la crisis de valores de la sociedad actual (Almacellas, 2013; Méndez-García, 2012). Estamos en una época en la que los que la sociedad y los medios de comunicación nos invitan a vivir el momento de la forma más placentera posible, sin pensar en las consecuencias de nuestros actos y a tomar decisiones encaminadas a la búsqueda del placer inmediato.

Sobre la actual crisis de valores numerosos autores consideran que ha habido un cambio de valores en las sociedades occidentales y una erosión en los mismos (Amuthavalli; Sridevi, 2014; Kumar, 2015; Pajaziti, 2014). Tal es así que la propia Organización de las Naciones Unidas para la Educación, la Ciencia y la Cultura (UNESCO) (1998) considera que la sociedad actual se encuentra inmersa en una profunda crisis de valores, centrados únicamente en la economía, por lo que considera que las instituciones educativas deben centrarse más en fomentar las dimensiones morales y espirituales con mayor arraigo.

Esta crisis de valores se centra más en aspectos éticos y morales que en aspectos intelectuales (Sharma, 2014), prevaleciendo valores como la riqueza, el orgullo, el egoísmo y la hipocresía. Así mismo, algunos autores (Garg, 2014; Sharma, 2014) consideran que la causa de la crisis de valores viene provocada por la alienación de la ciencia y la tecnología, que en vez de ayudar en el progreso de la humanidad se han convertido en una fuerza social de control de masas, contribuyendo también a la desaparición de los antiguos valores éticos y prosociales que garantizaban el bienestar del grupo. En esta misma línea, en muchas ocasiones, se considera que esta crisis de valores viene provocada por los medios tecnológicos que tenemos a nuestra disposición (Dong; Yi, 2015). Existen líneas de pensamiento que consideran que la TV y el cine nos han hecho ver como normales conductas que hace treinta y cinco años serían consideradas como intolerables (Pajaziti,2014), de tal manera que esa relajación de los valores morales han contribuido a numerosos conflictos éticos en la sociedad actual (Amuthavalli; Sridevi, 2014). Llevado al plano educativo se podría decir que muchos educadores a día de hoy consideran normales conductas que hace unos años serían consideradas como intolerables.

Amuthavalli y Sridevi (2014) consideran que la modernización de la sociedad actual ha cambiado nuestra forma de ver el mundo, de tal manera que los nuevos valores imperantes se centran en consumismo, el materialismo y el egoísmo. En muchas ocasiones alcanzar el éxito con el menor esfuerzo posible se ha convertido en la prioridad absoluta de numerosos jóvenes y adolescentes.

En este campo, el sistema de valores de Schwartz (1992) está gozando de un elevado auge (Cieciuch; Schwartz, 2012). Esta teoría acerca de los valores humanos ha sido probada en más de sessenta países (Schwartz; Boehnke, 2004; Schwartz et al., 2001) demostrando la existencia de diez valores perfectamente distinguibles que recogen las principales metas motivacionales de los seres humanos (Bilsky; Janik; Schwartz, 2011; Davidov, 2010). 
Los valores, desde la perspectiva de Schwartz, se definen como:

Creencias sobre estados, o conductas finales deseables, que trascienden a las situaciones específicas, que guían la selección o evaluación de la conducta y de los acontecimientos, y están ordenados según su importancia relativa. (Schwartz; Bilsky, 1987, p. 551)

En su teoría, Schwartz (1992) propone diez tipos de valores motivacionales en los seres humanos (logro, benevolencia, poder, universalismo, individualidad, hedonismo, tradición, seguridad, conformidad y estimulación) relacionados con las metas a las que se dirigen y los valores que se encuentran asociados (Cuadro 1). Los diez valores pueden ser replicados en culturas y grupos muy diferentes, y provienen de tres necesidades básicas de todo ser humano (Schwartz; Boehnke, 2004): las necesidades individuales como organismo biológico, las de interacción social coordinada y, finalmente, las de supervivencia y bienestar en los grupos.

Schwartz (1992) utilizó el escalamiento multidimensional para comprobar el ajuste de los datos empíricos a su teoría, ya que permite mostrar de forma simultánea las relaciones entre todos los valores en un espacio bidimensional (Schwartz, 2011). De esta forma, la estructura del modelo de valores se presenta en un circumplejo (Figura 1). Perrinjaquet et al. (2007) indican que este tipo de estructura puede representar variables espaciadas con igual tamaño u otras de tamaño desigual, según la importancia en la estructura. Con el mismo espacio entre las variables se denomina como modelo circulante y responde a la primera propuesta realizada por Schwartz (1992). La segunda propuesta, con el tamaño entre las variables desigual, se ha definido como modelo cuasi-circumplejo, siendo el que mejor se ajusta a la teoría de los valores humanos (Schwartz; Boehnke, 2004). La posición de los valores no es algo aleatorio, dado que la fuerza de asociación disminuye a medida que la distancia entre ellas es mayor, es decir, que cuanto mayor sea la distancia entre dos valores menor será la relación entre ambos (idem). Por ejemplo, acciones que expresen hedonismo probablemente entrarán en conflicto con aquellas acciones que expresen valores relativos a tradición; por el contrario, esas acciones serán compatibles con aquellas que expresen individualidad, como se representa en la Figura 1.

Las diferencias individuales de cada sujeto en los distintos valores vienen provocadas por una combinación personal de dotaciones biológicas, experiencias sociales y la exposición a las normas culturales que la sociedad considere como deseables (Roccas et al., 2002). En este sentido, el circumplejo se basa en las relaciones dinámicas existentes entre los valores, y la compatibilidad entre las metas motivacionales que subyacen de ellos es una de las características principales de la teoría (Schwartz; Boehnke, 2004). Estas relaciones dinámicas explican que la expresión de cualquiera de los valores tendrá consecuencias a diferentes niveles (psicológico, práctico y social) que pueden ser compatibles o no con la persecución de otros valores (Roccas et al., 2002; Schwartz; Bohenke, 2004; Schwartz; Sagiv; Boehnke, 2000).

Schwartz (1992) trata de explicar su modelo a partir de las interacciones que se pueden dar entre los valores. Todas estas relaciones se podrían sintetizar en una 


\section{Cuadro 1 - Definición de los tipos motivacionales de valores y los valores individuales que los representan}

\begin{tabular}{|c|c|c|}
\hline Valor & Meta final a la que se dirige & Valores representativos \\
\hline Poder & $\begin{array}{l}\text { Estatus social y prestigio, } \\
\text { control y dominancia sobre } \\
\text { la gente y los recursos. }\end{array}$ & $\begin{array}{l}\text { Autoridad, poder social, riqueza, } \\
\text { preservar mi imagen pública. }\end{array}$ \\
\hline Logro & $\begin{array}{l}\text { Éxito personal, demostrando } \\
\text { ser competente de acuerdo } \\
\text { con las normas sociales. }\end{array}$ & $\begin{array}{l}\text { Ambicioso, triunfador, } \\
\text { capaz, influyente. }\end{array}$ \\
\hline Hedonismo & Obtener placer para uno mismo. & $\begin{array}{l}\text { Placer, vida divertida, } \\
\text { autoindulgencia. }\end{array}$ \\
\hline Estimulación & Excitación, novedad desafíos en la vida. & $\begin{array}{l}\text { Una vida excitante, una vida } \\
\text { variada, atrevimiento. }\end{array}$ \\
\hline Individualidad & $\begin{array}{l}\text { Independencia en los pensamientos } \\
\text { y en las acciones, eligiendo, } \\
\text { creando, explorando. }\end{array}$ & $\begin{array}{l}\text { Creatividad, libertad, } \\
\text { independiente, escogiendo } \\
\text { sus propias metas, curioso. }\end{array}$ \\
\hline Universalismo & $\begin{array}{l}\text { Comprensión, aprecio, tolerancia } \\
\text { y protección del bienestar de las } \\
\text { personas y de la naturaleza. }\end{array}$ & $\begin{array}{l}\text { Igualdad, justicia social, sabiduría, } \\
\text { mente abierta, protección } \\
\text { del ambiente, unión con la } \\
\text { naturaleza, un mundo hermoso. }\end{array}$ \\
\hline Benevolencia & $\begin{array}{l}\text { Preservar y reforzar el bienestar } \\
\text { de la gente con la que se tiene un } \\
\text { contacto personal frecuente. }\end{array}$ & $\begin{array}{l}\text { Amabilidad, honestidad, } \\
\text { comprensivo, responsable, leal. }\end{array}$ \\
\hline Tradición & $\begin{array}{l}\text { Respeto, compromiso y aceptación de } \\
\text { las costumbres e ideas que propone } \\
\text { la tradición cultural o la religión. }\end{array}$ & $\begin{array}{l}\text { Devoto, respeto a la tradición, } \\
\text { moderado, humilde. }\end{array}$ \\
\hline Conformidad & $\begin{array}{l}\text { Moderación en las acciones, } \\
\text { inclinaciones e impulsos para no } \\
\text { ofender ni perjudicar a otros y violar } \\
\text { las expectativas sociales o las normas. }\end{array}$ & $\begin{array}{l}\text { Autodisciplina, educación, respetar } \\
\text { a padres y ancianos, obediencia. }\end{array}$ \\
\hline Seguridad & $\begin{array}{l}\text { Seguridad, armonía y estabilidad } \\
\text { de la sociedad, de las relaciones } \\
\text { y de uno mismo. }\end{array}$ & $\begin{array}{l}\text { Seguridad familiar, seguridad } \\
\text { nacional, orden social, } \\
\text { reciprocidad de favores. }\end{array}$ \\
\hline
\end{tabular}

Fuente: Adaptado de Schwartz, Sagiv y Boehnke (2000, p. 325-326).

Elaborado por los autores.

estructura bidimensional compuesta por cuatro tipos de orden superior (Figura 1), que combinan distintos tipos de valores:

1. Trascendencia: universalismo y benevolencia.

2. Promoción personal: poder y logro.

3. Conservación: seguridad, conformidad y tradición.

4. Apertura al cambio: estimulación e individualidad.

Hedonismo se encuentra entre los tipos de orden superior trascendencia y promoción personal, ya que comparte elementos de ambos. 


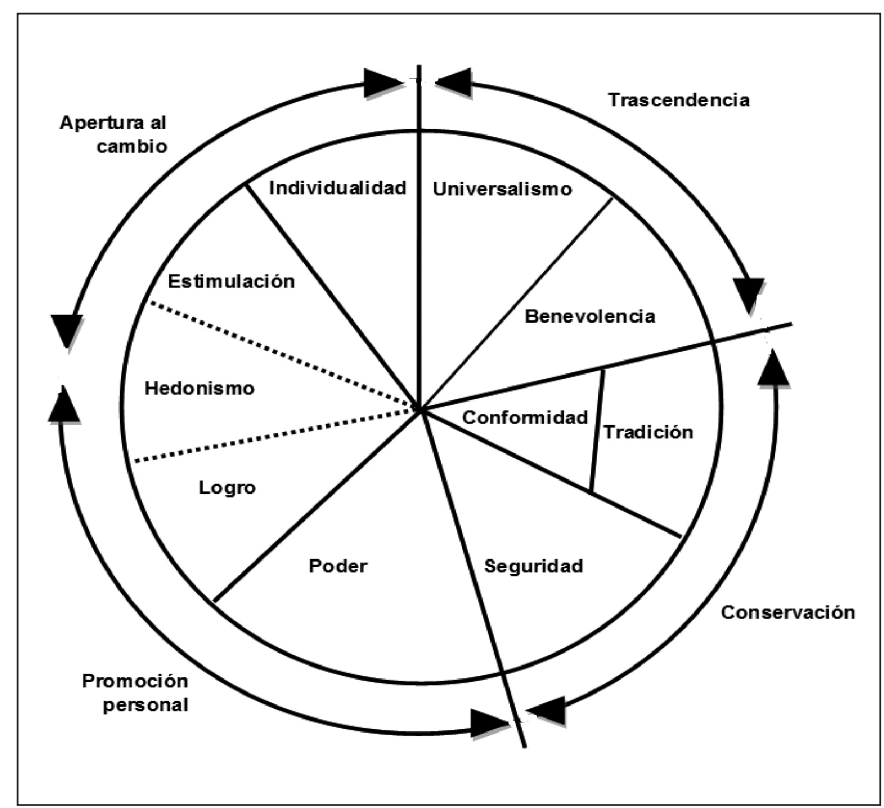

Figura 1 - Circumplejo de los valores de Schwartz. Fuente: adaptado de Schwartz y Boehnke (2004). Elaborado por los autores.

A pesar de que la intención inicial de Schwartz con respecto a estos tipos de orden superior era únicamente la de explicar la estructura de los valores de una manera más sencilla, en ocasiones se ha preferido utilizar estos cuatro tipos en vez de los diez valores con la idea de predecir algunas conductas y actitudes (Ros; Schwartz; Surkiss, 1999; Schwartz, 1994).

Schwartz y Boehnke (2004) asumen que los tipos de orden superior indicados poseen una mayor significación conceptual que cualquier otro tipo de combinación alternativa. Por lo tanto, se esperaría que valores adyacentes dentro de esos tipos de orden superior estuvieran asociados con mayor fuerza que con aquellos otros valores adyacentes pero que pertenecen a otros tipos de orden superior. Los resultados de los análisis factoriales confirmaron que el incremento de las intercorrelaciones entre los valores que se integraban dentro de los tipos de orden superior no produjo ninguna mejora en el ajuste del modelo, con lo cual se confirmó la idea de que los valores se encuentran formando un continuo motivacional. Esta idea implica que cualquier combinación de valores adyacentes puede ser formada en función de los intereses del investigador, siendo tan legítima como la propuesta anterior (idem).

La teoría de Schwartz se ha comprobado en diferentes países y culturas, como se ha indicado antes, casi siempre con sujetos adultos (Schwartz; Boehnke, 2004), siendo más raros los estudios realizados en adolescentes (Bubeck; Bilsky, 2004), niños (Döring, 2010; Döring et al., 2010) o personas mayores (García Maté, 2013). Este es el motivo por el cual nos preguntamos si el modelo es aplicable a la adoles- 
cencia, esto es, si entre los adolescentes se replica la estructura del circumplejo; si se producen diferencias de género; y qué implicaciones educativas podemos identificar con los resultados que obtengamos. Para responder a estos interrogantes, se plantea una investigación cuyos elementos constituyentes se describen a continuación.

\section{OBJETIVOS}

Comenzamos planteándonos unos objetivos acordes con las preguntas de investigación, es decir, nos proponemos estudiar la jerarquía de los valores humanos en adolescentes, comprobar si existen diferencias en función del género y plantear orientaciones educativas derivadas.

\section{MÉTODO}

\section{PARTICIPANTES}

La población total de alumnos que cursaban Educación Secundaria Obligatoria (ESO) y bachillerato durante el curso 2013-2014 en España era de un total de 2.524.053, siendo el porcentaje de varones de 50,27\% y el de mujeres de 50,73\%. $\mathrm{E}$ total de alumnos que cursaron ESO y bachillerato en la comunidad autónoma de Castilla y León, en la que se desarrolla el estudio fue de 182.600 (10\% del total de España). En nuestro estudio participaron en el estudio 499 adolescentes, 267 varones $(53,5 \%)$ y 232 mujeres $(46,5 \%)$ de diferentes centros escolares de educación secundaria de la comunidad de Castilla y León (España). El rango de edad comprende desde los 12 hasta los 18 años $(M=15,18 ; D T=1,75)$. Para la selección de los participantes, se contactó con diferentes centros educativos solicitando su colaboración en el proyecto. De este modo, la muestra tiene un carácter incidental, al estar compuesta por el alumnado de aquellos colegios que mostraron su interés en participar.

\section{INSTRUMENTO}

Se utilizó el cuestionario de valores personales (Anexo I) diseñado a partir del Schwartz Value Survey (Schwartz, 1992). En el cuestionario, los diez valores se evalúan con cinco ítems cada uno. Se optó por esta uniformidad siguiendo la sugerencia de Schwartz y Boehnke (2004), en la que indicaban que sería deseable estudiar cada valor con el mismo número de ítems para comprobar la estructura que encontramos en cada uno de ellos y en su conjunto. En el cuestionario original no todos los valores están representados por el mismo número de ítems, debido a que algunos son conceptualmente muy amplios (Schwartz, 1992).

Cada uno de los ítems se valora con una puntuación de 1 a 7 , siendo (1) “apenas o nada lo poseo", en referencia al valor enunciado, y aumentando el grado en que cree poseer el valor hasta llegar a (7) "lo poseo mucho o en alto grado". La puntuación total de cada valor se obtiene sumando los puntos de los cinco ítems. 
Datos sociodemográficos. Paralelamente se solicitaron un conjunto de datos sociodemográficos (edad, sexo, etc.), que permitieran un análisis más pormenorizado de los resultados.

\section{PROCEDIMIENTO}

Se solicitó consentimiento informado, para lo cual se informó adecuadamente a los centros participantes y progenitores del estudio que se deseaba realizar y todos ellos dieron su consentimiento.

Obtenida la respuesta afirmativa y los permisos necesarios, se estableció, de acuerdo con los propios centros, un calendario que redujera el impacto en las actividades de los mismos. En cada centro, los datos se recogieron en una única sesión de cincuenta minutos.

En la fase de aplicación se proporcionaron instrucciones verbales previas sobre cómo responder al cuestionario. En todos los casos los estudiantes debían responder a los cuestionarios dentro de su aula, supervisados por alguno de los responsables de la investigación asegurando el anonimato y la confidencialidad de las respuestas.

\section{ANÁLISIS DE DATOS}

En primer lugar, se estudiaron los índices de fiabilidad, por consistencia interna (Alfa de Cronbach), de las subescalas del cuestionario de valores personales. Para comprobar si en los adolescentes se replica la estructura del circumplejo propuesta por Schwartz se realizó una prueba de escalamiento multidimensional, siguiendo así los mismos pasos realizados originalmente por el autor. Posteriormente, se realizaron análisis descriptivos para explorar las preferencias de los valores en adolescentes. Se realizaron pruebas t para muestras independientes con la intención de determinar si existían diferencias significativas dentro de la jerarquía de valores en función del sexo. Previamente se contrastó que se cumplían los supuestos de homocedasticidad y normalidad.

El paquete estadístico utilizado fue el Statistica en su versión 7.0.

\section{RESULTADOS}

Los índices de fiabilidad obtenidos, por consistencia interna, para cada una de las subescalas del cuestionario, han sido satisfactorios. En todos los casos superaron un alfa de 0,60. Partiendo de que cada escala se compone de cinco ítems, fueron sólo tres subescalas las que no superaron un coeficiente de 0,70 : tradición $(0,64)$, logro $(0,67)$ e individualidad $(0,62)$. Si comparamos las fiabilidades obtenidas por los adolescentes con las obtenidas en otros estudios realizados con adultos (Olver; Mooradian, 2003; Luk; Bond, 1993), y teniendo en cuenta que en la mayoría de los casos el número de ítems difiere, se observa que los índices de fiabilidad han sido muy similares a los obtenidos por estos autores.

El escalamiento multidimensional nos permite obtener la estructura subyacente de los datos, pero la interpretación estadística cobra sentido a partir de la 
representación gráfica de las relaciones entre las variables. En nuestro caso fueron dos las dimensiones que mejor explicaban la configuración espacial, ya que para tal configuración se obtuvo un índice de stress $=0,08$, el cual indica el grado de ajuste entre el modelo y los datos. Cuanto más cercano es al valor a cero, más óptimo o adecuado es el ajuste (Young; Lewychyj, 1979).

En la Figura 2 se presenta la estructura subyacente obtenida para los diez valores personales. Tal y como se puede observar, la distribución espacial de los valores en los adolescentes se ajusta a la propuesta en el modelo teórico. Es decir, cada uno de los valores ocupa su lugar correspondiente. El orden de los valores a lo largo del circumplejo es idéntico al que se propone en el modelo teórico, si bien la distribución espacial se puede mejorar.

El análisis visual sirve para comprobar en la muestra de adolescentes los cuatro tipos de orden superior propuestos por Schwartz. La estructura bidimensional muestra los cuatro tipos de orden superior, es decir dos dimensiones bipolares, manteniendo el mismo orden y posición (Figura 2) al modelo del cuasi-circumplejo propuesto por Schwartz (Figura 1). En la parte superior izquierda se sitúan estimulación e individualidad, los cuales componen el tipo de orden superior apertura al cambio. El otro extremo de esta dimensión bipolar (abajo a la derecha) es el tipo conservación (compuesto por conformidad, tradición y seguridad). La dimensión promoción personal se encuentra ubicada en la parte superior derecha y en el extremo opuesto se sitúan los valores que integran el tipo de orden superior trascendencia. En la Figura 2 también se puede apreciar cómo en la parte superior derecha aparecen los dos valores que componen el tipo promoción personal, que son logro y poder. En su extremo opuesto, en la parte inferior a la izquierda, se sitúan los valores, universalidad y benevolencia, los cuales componen el polo opuesto, promoción personal.

Debemos llamar la atención sobre la situación que ocupa en el circumplejo el valor hedonismo en los adolescentes participantes. Schwartz (1992) ubicó este valor compartido entre apertura al cambio y promoción personal, pudiendo formar parte de los dos. En el caso de los adolescentes, encontramos un claro posicionamiento mucho más cercano al primer tipo de orden superior indicado, teniendo que realizar un esfuerzo teórico para que el ajuste sea perfecto respecto al modelo propuesto para los adultos.

En la Figura 3 se presentan, en orden descendente, las preferencias de los adolescentes a partir de las puntuaciones totales. El primer valor de la jerarquía para los adolescentes fue hedonismo. Es reseñable la importante diferencia de puntuación obtenida por Hedonismo respecto a las puntuaciones inmediatamente inferiores (estimulación e individualidad), y con respecto al valor con menor puntuación (poder). Es decir, la valoración de hedonismo se encuentra muy por encima que cualquier otro valor y, según Schwartz, ejercerá una gran influencia sobre los valores que se encuentran adyacentes en el circumplejo (estimulación y logro). Por tanto la influencia que hedonismo tendrá sobre estos dos valores es especialmente destacable en los adolescentes.

Los siguientes valores con mayor puntuación son estimulación e individualidad, que componen el tipo de orden superior apertura al cambio, seguido de los 


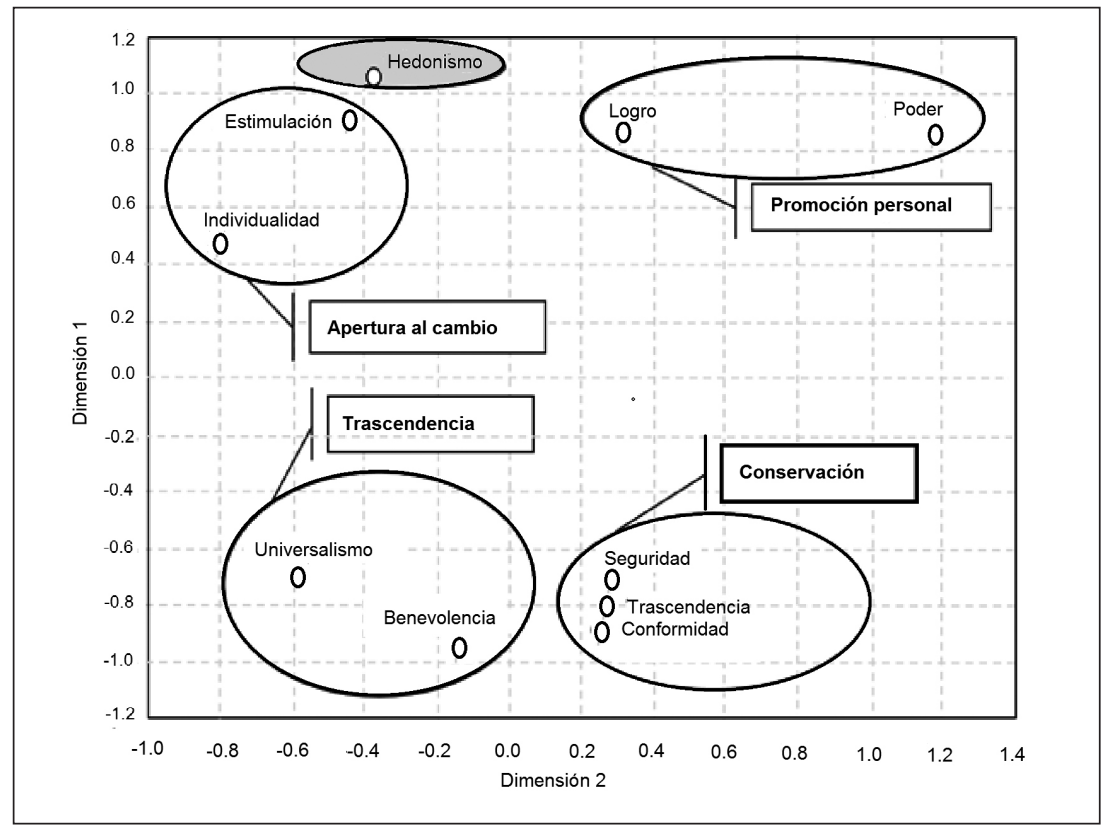

Figura 2 - Representación espacial de las relaciones entre los diez valores personales. Fuente: resultados de la investigación.

Elaborado por los autores.

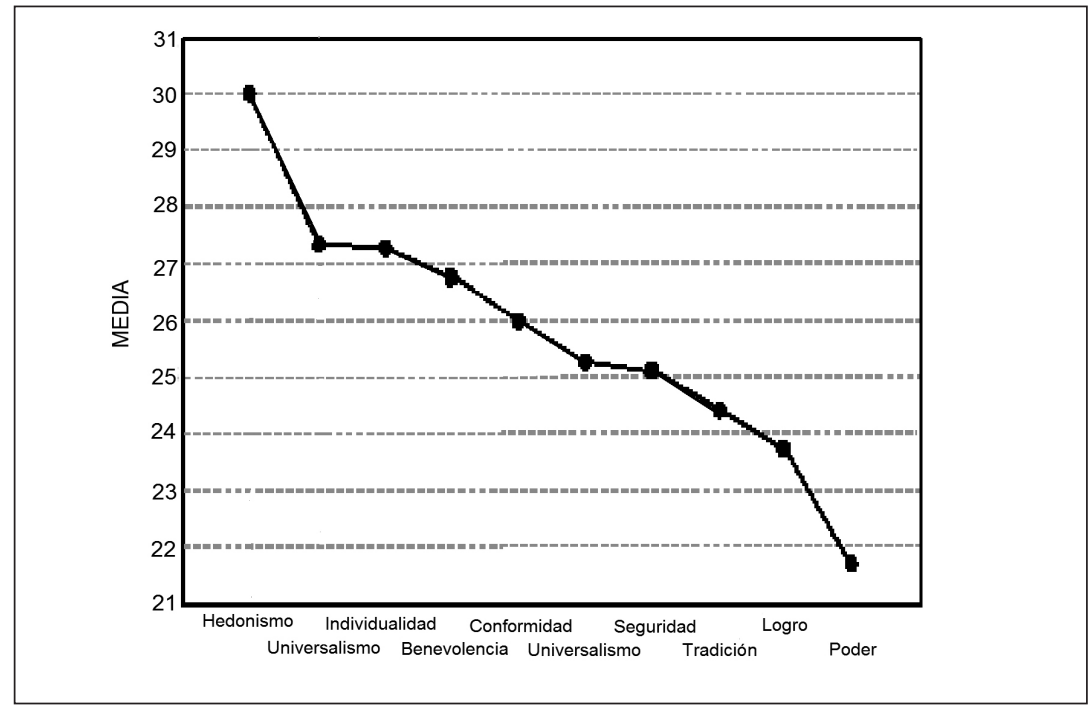

Figura 3 - Medias en orden descendente de los valores más preferidos a los menos preferidos en los adolescentes. Fuente: resultados de la investigación.

Elaborado por los autores. 
Cuadro 2 - Diferencias en las puntuaciones de los valores en función del sexo

\begin{tabular}{|c|c|c|c|c|c|c|}
\hline & \multicolumn{2}{|c|}{ Hombres } & \multicolumn{2}{|c|}{ Mujeres } & \multirow[b]{2}{*}{$\mathrm{t}(497)$} & \multirow[b]{2}{*}{$p$} \\
\hline & $M$ & SD & M & SD & & \\
\hline Logro & 24,57 & 4,98 & 24,24 & 5,12 & 0,73 & 0,46 \\
\hline Benevolencia & 26,68 & 5,15 & 28,96 & 4,76 & $-5,10$ & $<.00$ \\
\hline Poder & 22,78 & 6,27 & 21,85 & 5,99 & 1,68 & 0,094 \\
\hline Individualidad & 27,99 & 4,28 & 28,36 & 4,85 & $-0,91$ & 0,36 \\
\hline Universalismo & 25,47 & 5,86 & 26,68 & 6,11 & $-2,26$ & $<.02$ \\
\hline Tradición & 24,04 & 5,58 & 24,91 & 4,66 & $-1,85$ & 0,06 \\
\hline Seguridad & 24,50 & 5,67 & 25,84 & 4,83 & $-2,82$ & $<.00$ \\
\hline Estimulación & 27,35 & 5,43 & 27,48 & 5,60 & $-0,27$ & 0,79 \\
\hline Conformidad & 25,10 & 6,42 & 27,03 & 5,30 & $-3,61$ & $<.00$ \\
\hline Hedonismo & 30,03 & 4,19 & 29,91 & 4,99 & 0,29 & 0,77 \\
\hline
\end{tabular}

Fuente: resultados de la investigación.

Elaborado por los autores.

valores pertenecientes a trascendencia (universalismo y benevolencia), mientras que mostraron una menor preferencia por los opuestos a los anteriores en el circumplejo: conservación (seguridad, conformidad y tradición) y promoción personal (poder y logro).

Para estudiar las diferencias existentes por sexo, se utilizó la prueba t obteniendo los resultados que se recogen en la Cuadro 2.

En la Cuadro 2 se observa que las puntuaciones de las mujeres fueron superiores a las de los varones en 7 de los 10 valores. En el estudio comparado las pruebas $t$ realizadas revelaron diferencias significativas en función del sexo en cuatro de los diez valores analizados. En todos los casos las puntuaciones de las mujeres fueron superiores a las de los varones. En el tipo de orden superior trascendencia se obtuvieron diferencias significativas en los dos valores que lo integran, es decir en benevolencia $t(497)=-5,10, p<.00$ y universalismo $t(497)=-2,26, p=0,02$. También se obtuvieron diferencias en los valores seguridad $t(497)=-2,82, p=.00$ y conformidad $t(497)=-3,61, p<.00$, los cuales conforman junto con tradición el tipo de orden superior conservación. En el caso de tradición las mujeres obtuvieron mayor puntuación media que los varones, si bien esta diferencia no ha alcanzado a ser significativa $t(497)=-1,85, p=0,06$.

El valor preferido tanto por los varones como por las mujeres ha sido hedonismo, presentando unas puntuaciones casi idénticas en ambos sexos. En los tipos de orden superior, las mujeres dieron más importancia a los valores relacionados con trascendencia, mientras que los varones lo hicieron con los valores relacionados con promoción personal, tipo de orden superior opuesto. Otra diferencia fue que las mujeres obtuvieron una mayor puntuación en los valores que componen conservación, mientras que en su opuesto, apertura al cambio, las puntuaciones 
obtenidas por hombres y mujeres vuelven a ser muy similares. En ambos sexos se mostró mayor preferencia por los valores relacionados con trascendencia y apertura al cambio, y menor por aquellos relacionados con los tipos opuestos, promoción personal y conservación.

\section{DISCUSIÓN}

Para comprobar la estructura subyacente a los valores personales hemos utilizado el escalamiento multidimensional, comprobando que los valores forman un continuo motivacional entre los que existen una serie de relaciones dinámicas de congruencias e incongruencias en función de las metas que persigan cada uno de ellos. Para un mejor ajuste, sería adecuado que seguridad estuviera un poco más alejado de tradición y conformidad. Respecto a estos dos últimos valores, vemos cómo ambos se sitúan en el mismo plano angular, ocupando tradición un lugar un poco más periférico, lo cual es congruente con los resultados que Schwartz y Boehnke (2004) obtuvieron para la modificación del cuasi-circumplejo, en el que situaron tradición y conformidad en el mismo plano angular, pero el primero de ellos como periférico al segundo.

Hemos podido comprobar que la estructura del cuasi-circumplejo obtenida en nuestro estudio con adolescentes agrupa los valores según los cuatro tipos de orden superior propuestos en la teoría de Schwartz (Schwartz, 1992; Schwartz; Boehnke, 2004). Es decir, la dimensión compuesta por conservación/apertura al cambio se distribuye desde aquellos valores que motivan a las personas a seguir sus emociones e intereses intelectuales sin tener clara su dirección (apertura), hasta aquellos valores que implicarían la preservación del status quo y de la seguridad que esto proporciona (conservación). En la dimensión formada por los tipo de orden superior promoción personal/trascendencia se encuentran los valores que engloban las motivaciones que llevan a la gente a alcanzar sus propios objetivos sin importarles nada ni nadie (promoción personal), y por otro lado aquellos que de una manera no egoísta buscan el bienestar de los otros y de la naturaleza. Hedonismo se sitúa entre ambos tipos de orden superior puesto que comparte elementos de ambos.

La cercanía de Hedonismo a los valores de apertura al cambio (Figura 2) nos hace plantearnos la posibilidad de que para comprender mejor la estructura de valores de Schwartz en los adolescentes, esta categoría pudiera quedar compuesta por hedonismo, individualidad y estimulación; mientras que promoción personal lo formarían solamente logro y poder. La posición intermedia de hedonismo, entre dos tipos de orden superior, que se propone para los adultos no encuentra una clara correspondencia en los adolescentes participantes. Este planteamiento se formula al encontrar una enorme variación de este valor en función de la edad. En relación a esto último, nuestros resultados muestran que es el valor que consideran más importante, mientras que los adultos le dan una valoración intermedia (Schwartz; Bardi, 2001) y, en la tercera edad es el valor con menor puntuación (García Maté, 2013).

Como se ha referido, son pocos los estudios que han aplicado la teoría de Schwartz en adolescentes, por ejemplo Boehnke y Wezel (2006) evaluaron los valores propuestos por Schwartz en 121 niños con edades comprendidas entre 
los 7 y los 14 años, observando que las preferencias de los niños se estructuraban de acuerdo con los tipos de orden superior (trascendencia vs promoción personal; apertura al cambio vs conservación); sin embargo, los diez valores no aparecían estructurados como defiende la teoría de Schwartz. En nuestro caso, tanto la distribución espacial de los valores como la mostrada por los tipos de orden superior se ajustan a lo especificado por la teoría. Nuestros resultados, junto con los de otros estudios (Bubeck; Bilsky, 2004; Boehnke; Wezel, 2006), muestran que la teoría de los valores de Schwartz se puede aplicar a los adolescentes, sí bien se necesitan nuevas investigaciones que corroboren esta afirmación.

Schwartz y Bardi (2001), a partir de muestras de 61 países, propusieron una jerarquía de valores, en la que situaron benevolencia, universalismo e individualidad como los más importantes; siendo poder, tradición y estimulación los menos valorados; mientras que seguridad, conformidad, logro y hedonismo ocuparían posiciones intermedias. Esta estructura de valores en los adultos y la obtenida en nuestro estudio con adolescentes presentan importantes diferencias (Cuadro 3). Por ejemplo, observamos que los dos valores preferidos por los adolescentes, hedonismo y estimulación, ocupan posiciones inferiores en la jerarquía de los adultos. Poder y tradición se encuentran entre los últimos lugares en ambas jerarquías, mientras que sólo individualidad y conformidad ocupan lugares idénticos.

Comparando el orden de preferencia entre varones y mujeres, ambos grupos muestran su mayor valoración de hedonismo, como hemos indicado, siendo los menos valorados los incluidos en el tipo de orden superior promoción personal (logro y poder) y tradición. Varones y mujeres dan una alta a valoración al tipo apertura al cambio, lo cual supone una propuesta de valoración de las actividades

Cuadro 3 - Comparación de la jerarquía de valores de Schwartz y Bardi (2001) para adultos y el estudio actual con adolescentes

\begin{tabular}{|c|c|c|c|c|}
\hline \multicolumn{3}{|c|}{ Adolescentes } & \multirow{2}{*}{$\begin{array}{c}\text { Adultos } \\
\text { Schwartz y } \\
\text { Bardi (2001) }\end{array}$} & \multirow[t]{2}{*}{ Valoración } \\
\hline Mujeres & Varones & Ambos sexos & & \\
\hline Hedonismo & Hedonismo & Hedonismo & Benevolencia & \multirow{3}{*}{ Alta } \\
\hline Benevolencia & Individualidad & Estimulación & Universalismo & \\
\hline Individualidad & Estimulación & Individualidad & Individualidad & \\
\hline Estimulación & Benevolencia & Benevolencia & Seguridad & \multirow{4}{*}{ Media } \\
\hline Universalismo & Conformidad & Conformidad & Conformidad & \\
\hline Conformidad & Universalismo & Universalismo & Logro & \\
\hline Logro & Seguridad & Seguridad & Hedonismo & \\
\hline Seguridad & Tradición & Tradición & Poder & \multirow{3}{*}{ Baja } \\
\hline Tradición & Logro & Logro & Tradición & \\
\hline Poder & Poder & Poder & Estimulación & \\
\hline
\end{tabular}

Fuente: resultados de la investigación.

Elaborado por los autores. 
orientadas hacia el riesgo y las nuevas experiencias. Debemos tener en cuenta que los varones presentan un perfil que podríamos denominar como "puro", puesto que tras hedonismo, aparecen los valores que componen el tipo apertura al cambio.

En el caso de las mujeres, tanto benevolencia como universalismo, que constituyen el tipo de orden superior trascendencia, se sitúan en el nivel superior del sistema de valores. Para estos dos valores las puntuaciones de las mujeres son significativamente superiores a las de los varones. La presencia de dichos valores en la parte superior de la jerarquía puede suponer que se suavice el efecto de apertura al cambio por la inclusión del contexto social y familiar en el proceso de toma de decisiones. Es decir, mientras que los varones adolescentes priorizan el disfrute y la vivencia nuevas experiencias, las mujeres de esta misma edad tomarán las decisiones pensando en la familia y los amigos, lo cual puede amortiguar notablemente cualquier decisión que pueda poner en riesgo su futuro (personal, familiar, social o educativo), como así ponen de manifiesto algunos estudios (Martínez Baena et al., 2012).

En este sentido, estudios recientes sobre fracaso escolar (Fernández Enguita; Mena; Riviere, 2010) han indicado la necesidad de investigar si existe algo en el ámbito escolar que perjudique a los estudiantes masculinos, independientemente de su esfuerzo y de sus capacidades cognitivas. En este sentido es posible que la estructura de valores de los varones adolescentes, que aprecian en mayor medida el riesgo y asumen una importante carga de individualidad, reduciendo además la influencia de las personas que les rodean, pueda ser uno de los elementos que incidan negativamente en su promoción académica. La mediación de benevolencia, en el caso de las mujeres, puede convertirse en un factor de protección antes de tomar decisiones arriesgadas, así el interés por la satisfacción personal se verá reducido al pensar en las personas del entorno cercano.

La mayor importancia que los varones otorgan, en comparación con las mujeres, a promoción personal, y la mayor preferencia de éstas por a los valores relacionados con el tipo trascendencia y el valor seguridad es congruente con los hallazgos mostrados por Schwartz y Rubel (2005). Estos autores evaluaron las diferencias de sexo que podrían existir en la importancia que se concede a los valores, si bien también indicaron que estas diferencias no llegan a ser extremadamente importantes.

Los adolescentes han mostrado una clara preferencia por el valor hedonismo. Este hecho indica que el disfrute personal es el primer activador de sus motivaciones, potenciado porque los siguientes valores son los dos que componen el tipo de orden superior apertura al cambio (estimulación e individualidad); es decir, disfrutar buscando nuevos caminos, realizando actividades de estímulo, actividades nuevas, que supongan el disfrute a través del contacto con el riesgo, etc.

El diferente posicionamiento de estos mismos valores en los adultos (Schwartz; Boehnke, 2004), refleja la necesidad de ajustar adecuadamente las propuestas entre los jóvenes y sus educadores (progenitores y docentes), pues el natural pensamiento en una y otra edad puede conducir al conflicto intergeneracional. Mientras que los adolescentes colocan hedonismo en primer lugar, y a importante distancia el resto de valores, los adultos ubican este valor en el último escalón del 
nivel medio (Schwartz; Bardi, 2001) lo que nos lleva a pensar que pocos adultos, sin una adecuada reflexión o conocimientos sobre la adolescencia, pueden llegar a comprender que el mero disfrute sea la principal motivación de los mismos.

Así, los docentes y profesionales de la intervención social, que se encuentran implicados en el desarrollo de los adolescentes, reciben habitualmente formación sobre el proceso evolutivo, sus intereses, el desarrollo de la personalidad, etc.; pero también sería de gran interés que conocieran la diferente perspectiva que tienen con los valores adultos y adolescentes, dado que les permitiría valorar algunas respuestas que los adultos reciben de los adolescentes. En función de las preferencias de los adolescentes y según las definiciones de las metas motivacionales a las que se dirige cada valor (Schwartz; Sagiv; Bohenke, 2000), podemos interpretar que los riesgos que pueden asumir los adolescentes, para encontrar nuevos estímulos, se encuentran perfectamente identificados en su estructura de valores, ya que apertura al cambio (estimulación e individualidad) son los posicionados en los primeros niveles. Así mismo, la escasa valoración de tradición, logro y poder abrirá las puertas al intento de los adolescentes de encontrar nuevos caminos o nuevos retos que pongan en cuestión los aprendizajes propuestos por los docentes.

Por otro lado, la importante función educadora que debe cumplir la familia no siempre se encuentra acompañada con una información, y formación, adecuadas. Todo ello puede llevar a la incomprensión, debido a que a los adultos les puede resultar difícil apreciar los valores de cambio y las actitudes de riesgo, mientras que ellos priorizan benevolencia y universalismo (Schwartz; Bardi, 2001) escasamente valorados por los adolescentes. Los conflictos pueden aumentar si en la convivencia también se encuentran personas mayores, para quienes los valores considerados más importantes por los adolescentes se encuentran en posiciones muy inferiores (García Maté, 2013).

Cuando un adulto, desde su estructura de valores, desea exponer a los adolescentes las acciones que pueden abordar para mejorar su situación actual o futura es fácil que los segundos no compartan ni los objetivos, ni las propuestas para conseguirlos (Casco; Oliva, 2005). Teniendo en cuenta los valores prioritarios para los adolescentes, podemos pensar que las actividades más motivadoras para ellos serán aquellas que supongan riesgo y creatividad, y cuyo éxito requiera del estímulo individual (Oliva Delgado, 2007).

Es decir, aquellas que posibiliten romper con la rutina, la monotonía y la repetición de modelos. Las propuestas didácticas más adecuadas serían aquellas basadas en actividades de investigación, diseño y desarrollo de proyectos individuales y, sobre todo, grupales/colaborativas (Serrano-Cámara et al., 2014). En esta misma línea cabe pensar en un rechazo a aquel tipo de tareas o modelos que los adultos les planteen si, en opinión de los alumnos, limitan su capacidad creadora y su disfrute en el proceso. Nos referimos a la realización de resúmenes, redacciones, ejercicios repetitivos... Actividades que, en definitiva, son escasamente apreciadas por el alumnado adolescente desde su estructura de valores. Para superar este tipo de limitaciones, basándonos en la alta valoración de hedonismo y del tipo de orden superior apertura al cambio, podemos ofrecer alternativas más motivadoras como las mostradas en la Cuadro 4. 
Cuadro 4 - Propuestas para el cambio metodológico en el aula basadas en la estructura de valores de los adolescentes

\begin{tabular}{|l|l|}
\hline \multicolumn{1}{|c|}{ Propuesta tradicional } & \multicolumn{1}{c|}{ Propuesta motivadora } \\
\hline $\begin{array}{l}\text { Lectura de textos y } \\
\text { realización de resúmenes }\end{array}$ & $\begin{array}{l}\text { - Búsqueda de información en internet y } \\
\text { otras fuentes. } \\
\text { - Presentación y defensa de la información obtenida. } \\
\text { - Desarrollo de investigaciones sobre } \\
\text { temas espećficos. }\end{array}$ \\
\hline Redacciones & $\begin{array}{l}\text { - Elaboración de material en entornos multimedia. } \\
\text { - Utilización de wikis. } \\
\text { - Presentación en blogs. } \\
\text { - Coevaluación y comentario de los compañeros. }\end{array}$ \\
\hline Ejercicios repetitivos & $\begin{array}{l}\text { - Actividades colaborativas de corrección } \\
\text { de ejercicios a través de internet. } \\
\end{array}$ \\
$\begin{array}{l}\text { - Proyectos de trabajo colaborativos. } \\
\text { - Aprendizaje basado en proyectos. } \\
\text { - Problemas cuya resolución no sea única. }\end{array}$ \\
\hline Aprendizajes memorísticos & $\begin{array}{l}\text { - Relación con el contexto a través de } \\
\text { búsquedas activas del alumnado. }\end{array}$ \\
\hline
\end{tabular}

Fuente: resultados de la investigación.

Elaborado por los autores.

Todas las propuestas en las que medie internet (Saeed; Yang; Sinnappan, 2009), y especialmente con el uso de las redes sociales (Abella; Delgado, 2012), entroncan directamente con su estructura de valores, y pueden ser identificadas por los adolescentes como propuestas novedosas (Compagnucci; Cardós, 2002), dado que incorpora recursos que prioritariamente utilizan para su ocio (hedonismo) o les permite percibirse como creadores o buscadores en un medio de gran valor para ellos (estimulación) (Buckingham; Martínez-Rodríguez, 2013). Así mismo, la intervención entre iguales, con una estructura de valores comunes, puede facilitar el entendimiento y mediación en situaciones de conflicto entre adolescentes $\mathrm{u}$ profesorado (Dekovic, 2007) y serán percibidas como de gran interés por su parte.

Todas estas propuestas se realizan con la intención de frenar las elevadas tasas de abandono escolar prematuro (Comisión Europea, 2011) que pueden estar relacionadas por el choque entre la estructura de valores de unos y las metodologías didácticas de otros.

\section{CONCLUSIONES Y PROSPECTIVA}

En este estudio hemos tratado de identificar el orden de preferencia de una muestra adolescentes (12-18 años) de los valores propuestos por Schwartz (1992) en su teoría de los valores humanos básicos, comprobar si existen diferencias significativas en función del sexo y proponer medidas educativas consecuentes con sus valores.

Encontramos que los adolescentes cuentan con una estructura de valores sensiblemente diferente a la de los adultos. El valor que obtiene mayor puntuación 
es el hedonismo, que se propone como elemento activador de los intereses de los adolescentes, seguido por los relacionados con la apertura al cambio (estimulación e individualidad).

Las diferencias encontradas con los adultos, revela la especial atención que deben poner los docentes en la planificación de las actividades. Propuestas educativas que el alumnado identifique como estimuladoras, que desarrollen sus capacidades, serán muy valorados por el ellos en estas edades. Es en ese contexto motivador donde internet se visualiza, a través de herramientas colaborativas y actividades de estímulo hacia la investigación, como un elemento que debe ser propiciado en el desarrollo de la actividad docente.

Los resultados de nuestro estudio plantean la necesidad de incluir en el plan de estudios de educación secundaria y bachillerato una asignatura obligatoria para educar en valores humanos. Consideramos que no son adecuadas políticas educativas basadas en la economía y el emprendimiento, como la Ley Orgánica para la Mejora de la Calidad Educativa (LOMCE) (Ministerio de Educación, Cultura y Deporte, 2013), ya que la primacía de valores como hedonismo se han dado por el desarrollo económico insostenible y las infinitas posibilidades de ocio y diversión a las que supuestamente no se pueden renunciar (Almacellas, 2013), por lo que deben anteponerse a valores como logro cuya meta es alcanzar el éxito, pero de acuerdo con las normas sociales. La LOMCE indica que los valores se trabajarán de forma transversal en todas las materias, dando a entender que los valores no se pueden enseñar como el resto de las materias sino que se trasmiten de forma indirecta en la convivencia escolar. Este enfoque no es el adecuado en una sociedad plural, ya que si bien cada materia incluye una serie de valores que deben ser enseñados a los alumnos se hace necesaria una asignatura que permita, desde la individualidad de cada sujeto, la construcción de un sistema de valores desde una mirada crítica, creativa y transformadora (Martín; Puig, 1998). Se hace necesaria entonces una revisión de los valores que priman en el sistema educativo, ya que no todo consiste en reflotar la economía sino que es necesario formar en valores cuyas metas motivacionales favorezcan la convivencia en grupo, la solidaridad, el respeto, el esfuerzo, etc.

Las diferencias de género también aparecen como un factor de interés. Mientras ellos buscarán en mayor medida el placer y el estímulo, en ellas mediará en sus actos la valoración de que sus actividades no supongan un daño a la familia. Estas diferencias, gestionadas adecuadamente por los docentes, permitirán ayudar a reducir las situaciones de conflicto o a estimular las propuestas para conseguir que los jóvenes puedan llegar a los objetivos más altos.

Finalmente, abrimos la puerta a futuras líneas de investigación que traten de explicar si las diferencias encontradas entre varones y mujeres corresponden realmente al sexo o tienen mucho que ver la influencia de la cultura a través de los estereotipos relacionados con esa variable. Consideramos también necesarios estudios evolutivos que analicen los cambios en la estructura de los valores en ambos sexos a lo largo de diferentes edades, y no sólo eso, sino que también comparen la evolución de la importancia que se da a los valores en las diferentes etapas del ciclo vital, como el realizado por Lyons, Duxbury y Higgins (2007). 


\section{REFERENCIAS}

Abella, V.; Delgado, V. Aplicación educativa de Twitter en la formación inicial del profesorado.Jornadas Universitarias de Tecnología Educativa, 20., 2012. Girona, España. Anais... Girona, 28-29 junio, 2012.p. 260-266.

Almacellas, M. Á. Salir del laberinto de la profunda crisis de valores. International Studies on Law and Education, Oporto: Editora Mandruvá, v. 13/14, p.111-120, enero/ agosto 2013.

Amuthavalli, T.; Sridevi, M. A study of the value orientation of degree college students. MIER Journal of Educational Studies, Trends E Practices, Jammu: Model Institute of Education \& Research, v. 4, n. 2, p. 262-271, Nov. 2014.

Bils ky, W.; Janik, M.; Schwartz, S. H. The structural organization of human values: evidence from three rounds of the European Social Survey (ESS). Journal of CrossCultural Psychology, Bellingham: IACCP; Thousand Oaks: Sage, v. 42, n. 5, p. 759-776, July 2011.

Boennke, K.; Wezel, C. Value transmission and value change: an exploratory threegeneration study. Zeitschrift fur Soziologie der Erziehung und Sozialisation, Frankfurt am Main: DIPF, Pedocs, v. 26, n. 4, p. 341-360, 2006.

Bubeck, M.; Bilsky, W. Value structure at early age. Swiss Journal of Psychology, Berna: Verlag Hans Huber, v. 63, n. 1, p. 31-41, 2004.

Buckingham, D.; Martínez-Rodríguez, J. B. Jóvenes interactivos: nueva ciudadanía entre redes sociales y escenarios escolares. Comunicar, Huelva: Grupo Comunicar, v. 40, n. 20, p. 10-13, marzo 2013.

Casal,J.; Merino, R.; García, M. Pasado y futuro del estudio sobre la transición de los jóvenes. Papers: Revista de Sociología, Barcelona: Universidad Autónoma de Barcelona, v. 96, n. 4, p. 1.139-1.162, 2011.

Casco, F. J.; Oliva, A. Valores y expectativas sobre la adolescencia: discrepancias entre padres, profesores, mayores y adolescentes. Infancia y Aprendizaje, Barcelona: Fundación Infancia y Aprendizaje, Taylor \& Francis, v. 28, n. 2, p. 209-222, 2005.

Cieciuch, J.; Schwartz, S. H. The number of distinct values and their structure assessed by PV-40. Journal of Personality Assessment, London: Routledge, v. 94, n. 3, p. 321-328, Feb. 2012.

Comisión Europea. Abordar el abandono escolar prematuro: una contribución clave al Consejo, al Comité Económico y Social Europeo y al Comité de las Naciones. Bruselas, Comisión Europea, 2011. Disponible en: <http://www.europarl.europa.eu/sides/getDoc. do?pubRef=-//EP//TEXT+REPORT+A7-2011-0363+0+DOC+XML+V0//ES>. Consultado el: 22 abr. 2014.

Compagnucci, E.; Cardós, P. El adolescente frente al conocimiento. Orientación y Sociedad, La Plata: Facultad de Psicología, 2002. v. 3.

DAvidov, E. Testing for comparability of human values across countries and time with the third round of the European Social Survey. International Journal of Comparative Sociology, Thousand Oaks: Sage, v. 51, n. 3, p. 171-191, June 2010. 
Deкоvic, M. Discrepancies between parental and adolescent developmental expectations. Conferencia de la Sociedad para la Investigación de la Adolescencia, 9., 2002, Luisiana, Nueva Orleans. Anais... Luisiana, Abr. 2002. p. 11-14.

Dong, G.; YI, L. Y. Soul confusion and moral education of human beings in technology age. Higher Education of Social Science, Québec: Canadian Research \& Development Center of Sciences and Cultures, v. 8, n. 1, p. 82-86, Jan. 2015.

Döring, A. K. Assessing childrens's values: an exploratory study. Journal of Psychoeducational Assessment, Thousand Oaks: Sage, v. 28, n. 6, p. 564-577, Jan. 2010.

.; Blauensteiner, A.; Aryus, K.; Drögekamp, L.; Bilsky, W. Assessing values at an early age: the Picture-Based Value Survey for Children (PBVS-C). Journal of Personality Assessment, London: Routledge, v. 92, n. 5, p. 439-448, Aug. 2010.

Fernández Enguita, M.; Mena, L.; Riviere, J. Fracaso y abandono escolar en España. Barcelona: Obra social La Caixa, 2010. (Colección Estudios Sociales, n. 29). Disponible en: <http://multimedia.lacaixa.es/lacaixa/ondemand/obrasocial/pdf/estudiossociales/ vol29_completo_es.pdf>. Consultado el: 18 mayo 2014.

García Maté, E. Diseño y evaluación de un programa de entrenamiento en valores como variable moduladora de la autorregulación en personas mayores universitarias. 2013. 342p. Tesis (Doctorado en Ciencias de la Educación) - Universidad de Burgos, Burgos, 2013. GARG, A. Value conflict and aggression among adolescents in relation to family climate. International Journal of Education, Meerut: Vidya Publications, v. 3, p. 15-24, dic. 2014. Kumar, V. Essential principles for quality education. International Journal of Multidisciplinary Educational Research, Visakhapatnam: Sucharitha Publications, v. 4, n. 5, p. 19-29, May 2015.

Luk, C. L.; Bond, M. H. Personality variations and values endorsement in Chinese university students. Personality and Individual Differences, United Kingdom: Elsevier, v. 14, n. 3, p. 429-437, Mar. 1993.

Lyons, S. T.; Duxbury, L.; Higgins, C. An empirical assessment of generational differences in basic human values. Psychological Reports, Missoula: Ammons Scientific, v. 101, n. 2, p. 339-352, Oct. 2007.

Martín, X.; Puig, J. M. Las siete competencias básicas para educar en valores. 1. ed. Barcelona: Graó, 2007.

Martínez Baena, A. C.; Chillón, P.; Martín-Matillas, M.; Pérez López, I.; Castillo, R.; Zapatera, B. et al. Motivos de abandono y no actividad físico-deportiva en adolescentes españoles: estudio Avena. Cuadernos de Psicología del Deporte, Murcia: Servicio de Publicaciones de la Universidad de Murcia, v. 12, n. 1, p. 45-54, jun. 2012. Méndez-García, J. M. ¿Crisis económica o crisis de valores? Una propuesta axiológica. 1. ed. Madrid: Sepha, 2012.

Ministerio de Educación, Cultura y Deporte. Ley Orgánica para la Mejora de la CalidadEducativa. Madrid: BOE, 2013. Disponible en: <http://www.boe.es/diario_boe/ txt.php?id=BOE-A-2013-12886>. Consultado el: 8 sept. 2016. 
Oliva Delgado, A. Desarrollo cerebral y asunción de riesgos durante la adolescencia. Apuntes de Psicología, Sevilla: Colegio Oficial de Psicología de Andalucía Occidental y Universidad de Sevilla, v. 25, n. 3, p. 239-254, 2007.

Olver, J.; Mooradian, T. Personality traits and personal values: a conceptual and empirical integration. Personality and Individual Differences, United Kingdom: Elsevier, v. 35, n. 1, p. 109-125, July 2003.

Pajaziti, A. Values and academia: a sociological-empirical approach. Revue des Sciences Politiques, Craiova: Editora Universitaria Craiova, v. 43, p. 125-140, 2014.

Perrinjaquet, A.; Furrer, O.; Usunier,J.-C.; Cestre, G.; Valette-Florence, P. A test of the quasi-circumplex structure of human values. Journal of Research in Personality, Amsterdam: Elsevier, v. 41, n. 4, p. 820-840, Aug. 2007.

Roccas, S.; Sagiv, L.; Schwartz, S. H.; Knafo, A. The Big Five personality factors and personal values. Personality and Social Psychology Bulletin, Thousand Oaks: Sage, v. 28, n. 6, p. 789-801, June 2002.

Ros, M.; Schwartz, S. H.; Surkiss, S. Basic individual values, work values and the meaning of work. Applied Psychology: An International Review, United Kingdom: John Wiley \& Sons, v. 48, n. 1, p. 49-71, Jan. 1999.

SAEEd, N.; YAng, Y.; Sinnappan, S. Emerging web technologies in higher education: a case of incorporating blogs, podcasts and social bookmarks in a web programming course based on students' learning styles and technology preferences. Educational Technology E' Society, Atenas: IFETS, v. 12, n. 4, p. 98-109, Oct. 2009.

Schwartz, S. H. Universals in the content and structure of values: theory and empirical tests in 20 countries. Advances in Experimental Social Psychology, New York: Academic Press, v. 25, p. 1-65, 1992.

Are there universal aspects in the content and structure of the values? Journal of Social Issues, Washington: SPSSI; United Kingdom: John Wiley \& Sons, v. 50, n. 4, p. 19-45, 1994.

. Studying values: personal adventure, future directions. Journal of Cross-Cultural Psychology, Thousand Oaks: Sage, v. 42, n. 2, p. 307-319, Mar. 2011.

;; BARDI, A. Value hierarchies across cultures: taking a similarities perspective. Journal of Cross-Cultural Psychology, Bellingham: IACCP; Thousand Oaks: Sage, v. 32, n. 3, p. 268-390, May 2001.

; Boemnke, K. Evaluating the structure of human values with confirmatory factor analysis. Journal of Research in Personality, Amsterdam: Elsevier, v. 38, n. 3, p. 230-255 , June 2004.

.; Bilsky, W. Toward a universal psychological structure of human values. Journal of Personality and Social Psychology, Washington, DC: American Psychological Association, v. 53, n. 3, p. 550-562, 1987.

.; Melech, G.; Lehmann, A.; Burgess, S.; Harris, M.; Owens, V. Extending the cross-cultural validity of the theory of basic human values with a different method of measurement. Journal of Cross-Cultural Psychology, Bellingham: IACCP; Thousand Oaks: Sage, v. 32, n. 5, Sept. 2001. 
;; Sagiv, L.; Boennke, K. Worries and values. Journal of Personality, Oxford: Blackwell Publishers, v. 68, n. 2, p. 309-346, Apr. 2000.

.; Rubel, T. Sex differences in value priorities: cross-cultural and multimethod studies. Journal of Personality and Social Psychology, Washington, DC: American Psychological Association, v. 89, n. 6, p. 1.010-1.028, Dec. 2005.

Serrano-Cámara, L. M.; Paredes-Velasco, M; Alcover, C. M.; VelázquezItURBide, J. Á. An evaluation of students' motivation in computer-supported collaborative learning of programming concepts. Computers in Human Behavior, Amsterdam: Elsevier, v. 31, p. 499-508, Feb. 2014.

Sharma, N. Value crisis: a global phenomenon. Asian Journal of Multidisciplinary Studies, Pune: Sonhira Publications, v. 2, n. 9, p. 66-69, Sept. 2014.

Unesco - Organización de las Naciones Unidas para la Educación, la Ciencia y la Cultura. Declaración Mundial sobre la Educación Superior en el Siglo XXI: visión y acción. París, UNESCO, 1998. Disponible en: <http://www.unesco.org/education/ educprog/wche/declaration_spa.htm>. Consultado el: 8 sept. 2016.

Young, F. W.; Lewychyj, R. ALSCAL User's guide. 3. ed. Chapel Hill: Data Analysis and Theory Associates, 1979. 


\section{ANEXO I}

\section{Cuestionario de Valores Personales}

Nombre y apellidos

Sexo (varón) $\ldots \ldots \ldots$ (mujer) $\ldots \ldots \ldots$

Instrucciones. ¿Cómo calificarías el grado en el que posees los siguientes valores?

Rodea con un círculo el número que mejor refleja el grado en que posees el valor enunciado en cada uno de los 50 ítems $(25+25)$.

Los extremos de la escala son el 1 y el 7, significando el 1 "apenas o nada lo poseo" y aumentando numéricamente el grado de tu estimación hasta el 7 "lo poseo mucho o en alto grado".

Gracias

\begin{tabular}{|l|l|l|l|l|l|l|l|}
\hline 1. Tener ambición & 1 & 2 & 3 & 4 & 5 & 6 & 7 \\
\hline 2. Ayudar a otros & 1 & 2 & 3 & 4 & 5 & 6 & 7 \\
\hline 3. Poseer autoridad & 1 & 2 & 3 & 4 & 5 & 6 & 7 \\
\hline 4. Eligiendo mis propias metas & 1 & 2 & 3 & 4 & 5 & 6 & 7 \\
\hline 5. Un mundo hermoso & 1 & 2 & 3 & 4 & 5 & 6 & 7 \\
\hline 6. Con capacidad & 1 & 2 & 3 & 4 & 5 & 6 & 7 \\
\hline 7. Perdonar & 1 & 2 & 3 & 4 & 5 & 6 & 7 \\
\hline 8. Preservar mi imagen pública & 1 & 2 & 3 & 4 & 5 & 6 & 7 \\
\hline 9. Creatividad & 1 & 2 & 3 & 4 & 5 & 6 & 7 \\
\hline 10. Protección del medio & 1 & 2 & 3 & 4 & 5 & 6 & 7 \\
\hline 11. Influyente & 1 & 2 & 3 & 4 & 5 & 6 & 7 \\
\hline 12. Honestidad & 1 & 2 & 3 & 4 & 5 & 6 & 7 \\
\hline 13. Poder social & 1 & 2 & 3 & 4 & 5 & 6 & 7 \\
\hline 14. Curiosidad & 1 & 2 & 3 & 4 & 5 & 6 & 7 \\
\hline 15. Justicia social & 1 & 2 & 3 & 4 & 5 & 6 & 7 \\
\hline 16. Ser inteligente & 1 & 2 & 3 & 4 & 5 & 6 & 7 \\
\hline 17. Lealtad & 1 & 2 & 3 & 4 & 5 & 6 & 7 \\
\hline 18. Reconocimientos social & 1 & 2 & 3 & 4 & 5 & 6 & 7 \\
\hline 19. Libertad & 1 & 2 & 3 & 4 & 5 & 6 & 7 \\
\hline 20. Unión con la naturaleza & 1 & 2 & 3 & 4 & 5 & 6 & 7 \\
\hline 21. Lograr éxito & 1 & 2 & 3 & 4 & 5 & 6 & 7 \\
\hline 22. Ser responsable & 1 & 2 & 3 & 4 & 5 & 6 & 7 \\
\hline 23. Conseguir riqueza & 1 & 2 & 3 & 4 & 5 & 6 & 7 \\
\hline 24. Independiente & 1 & 2 & 3 & 4 & 5 & 6 & 7 \\
\hline 25. Conocimiento del mundo & 1 & 2 & 3 & 4 & 5 & 6 & 7 \\
\hline & & & & & \\
\hline & 1 & & & \\
\hline
\end{tabular}




\section{Segunda parte del Cuestionario de Valores Personales}

\begin{tabular}{|c|c|c|c|c|c|c|c|}
\hline 1. Respetar la tradición & 1 & 2 & 3 & 4 & 5 & 6 & 7 \\
\hline 2. Seguridad familiar & 1 & 2 & 3 & 4 & 5 & 6 & 7 \\
\hline 3. Una vida variada & 1 & 2 & 3 & 4 & 5 & 6 & 7 \\
\hline 4. Obediencia & 1 & 2 & 3 & 4 & 5 & 6 & 7 \\
\hline 5. Obtener placer & 1 & 2 & 3 & 4 & 5 & 6 & 7 \\
\hline 6. Aceptar mi parte de la vida & 1 & 2 & 3 & 4 & 5 & 6 & 7 \\
\hline 7. Seguridad nacional & 1 & 2 & 3 & 4 & 5 & 6 & 7 \\
\hline 8. Una vida excitante & 1 & 2 & 3 & 4 & 5 & 6 & 7 \\
\hline 9. Honrar padres y ancianos & 1 & 2 & 3 & 4 & 5 & 6 & 7 \\
\hline 10. Disfrutar la vida & 1 & 2 & 3 & 4 & 5 & 6 & 7 \\
\hline 11. Devociones & 1 & 2 & 3 & 4 & 5 & 6 & 7 \\
\hline 12. Orden social & 1 & 2 & 3 & 4 & 5 & 6 & 7 \\
\hline 13. Atrevimiento & 1 & 2 & 3 & 4 & 5 & 6 & 7 \\
\hline 14. Autodisciplina & 1 & 2 & 3 & 4 & 5 & 6 & 7 \\
\hline 15. Concederse satisfacciones & 1 & 2 & 3 & 4 & 5 & 6 & 7 \\
\hline 16. Ser humilde & 1 & 2 & 3 & 4 & 5 & 6 & 7 \\
\hline 17. Jugar limpio & 1 & 2 & 3 & 4 & 5 & 6 & 7 \\
\hline 18. Buscar nuevas sensaciones & 1 & 2 & 3 & 4 & 5 & 6 & 7 \\
\hline 19. Buenos modales & 1 & 2 & 3 & 4 & 5 & 6 & 7 \\
\hline 20. Gozar al máximo ser moderado & 1 & 2 & 3 & 4 & 5 & 6 & 7 \\
\hline 21. Ser moderado & 1 & 2 & 3 & 4 & 5 & 6 & 7 \\
\hline 22. Reciprocidad de favores & 1 & 2 & 3 & 4 & 5 & 6 & 7 \\
\hline 23. Intentar retos desafiantes & 1 & 2 & 3 & 4 & 5 & 6 & 7 \\
\hline 24. Normas de convivencia & 1 & 2 & 3 & 4 & 5 & 6 & 7 \\
\hline 25. Diversiones & 1 & 2 & 3 & 4 & 5 & 6 & 7 \\
\hline
\end{tabular}

\begin{tabular}{|l|l|l|l|l|}
\hline \multicolumn{5}{|c|}{ AUTO-CORRECCION (Puestos abajo los puntos de cada ítem, se suman las columnas) } \\
\hline \multicolumn{1}{|c|}{ T } & \multicolumn{1}{c|}{ S } & \multicolumn{1}{c|}{ E } & \multicolumn{1}{c|}{ C } & \multicolumn{1}{c|}{ H } \\
\hline Ítem $1=$ & Ítem $2=$ & Ítem $3=$ & Ítem $4=$ & Ítem $5=$ \\
\hline Ítem $6=$ & Ítem $7=$ & Ítem $8=$ & Ítem $9=$ & Ítem $10=$ \\
\hline Ítem $11=$ & Ítem $12=$ & Ítem $13=$ & Ítem $14=$ & Ítem $15=$ \\
\hline Ítem $16=$ & Ítem $17=$ & Ítem $18=$ & Ítem $19=$ & Ítem $20=$ \\
\hline Ítem $21=$ & Ítem $22=$ & Ítem $23=$ & Ítem $24=$ & Ítem $25=$ \\
\hline Total $\mathrm{T}=$ & Total $\mathrm{S}=$ & Total $\mathrm{E}=$ & Total $\mathrm{C}=$ & Total $\mathrm{H}=$ \\
\hline
\end{tabular}




\begin{tabular}{|l|l|l|l|l|}
\hline \multicolumn{5}{|c|}{ AUTO-CORRECCION (Puestos abajo los puntos de cada ítem, se suman las columnas) } \\
\hline \multicolumn{1}{|c|}{$\mathrm{L}$} & \multicolumn{1}{c|}{$\mathrm{B}$} & \multicolumn{1}{c|}{$\mathrm{P}$} & \multicolumn{1}{c|}{$\mathrm{I}$} & \multicolumn{1}{c|}{$\mathrm{U}$} \\
\hline Ítem $1=$ & Ítem $2=$ & Ítem $3=$ & Ítem $4=$ & Ítem $5=$ \\
\hline Ítem $6=$ & Ítem $7=$ & Ítem $8=$ & Ítem $9=$ & Ítem $10=$ \\
\hline Ítem $11=$ & Ítem $12=$ & Ítem $13=$ & Ítem $14=$ & Ítem $15=$ \\
\hline Ítem $16=$ & Ítem $17=$ & Ítem $18=$ & Ítem $19=$ & Ítem $20=$ \\
\hline Ítem $21=$ & Ítem $22=$ & Ítem $23=$ & Ítem $24=$ & Ítem $25=$ \\
\hline Total $\mathrm{L}=$ & Total $\mathrm{B}=$ & Total $\mathrm{P}=$ & Total $\mathrm{I}=$ & Total $\mathrm{U}=$ \\
\hline
\end{tabular}

\section{SOBRE LOS AUTORES}

Víctor Abella García es doctor en psicología y ciencias de la educación por la Universidad de León (España). Profesor de la Universidad de Burgos (España).

E-mail: vabella@ubu.es

Fernando Lezcano Barbero es doctor en ciencias de la educación por la Universidad de Burgos (España). Profesor de la misma institución. E-mail: flezcano@ubu.es

Raquel Casado Muñoz es doctora en ciencias de la educación por la Universidad de Burgos (España). Profesora de la misma institución. E-mail: rcasado@ubu.es

Recebido em 12 de setembro de 2014 Aprovado em 17 de agosto de 2015 